\title{
Depth estimation of the absorbing structure in a slab turbid medium using point spread function
}

\author{
Ngoc An Dang Nguyen ${ }^{1}$, To Ni Phan Van² ${ }^{2}$ Kohei Yamamoto ${ }^{3}$, Minh Quang Nguyen ${ }^{1}$, Anh Tu Tran ${ }^{1}$, \\ Takeshi Namita ${ }^{4}$, Koichi Shimizu ${ }^{2}$, Trung Nghia Tran ${ }^{1, *}$
}

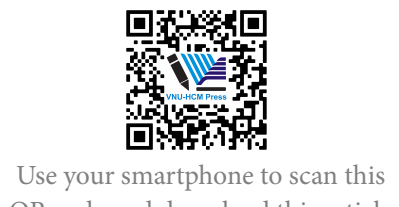

QR code and download this article

${ }^{1}$ Ho Chi Minh City University of Technology, VNU-HCM, Vietnam

${ }^{2}$ Waseda University, Kitakyushu, 808-0135 Japan

${ }^{3}$ Hokkaido University, Sapporo, 060-0814 Japan

${ }^{4}$ Kyoto University, Kyoto, 606-8507 Japan

Correspondence

Trung Nghia Tran, Ho Chi Minh City University of Technology, VNU-HCM, Vietnam

Email: ttnghia@hcmut.edu.vn

History

- Received: 08-3-2020

- Accepted: 15-10-2020

- Published: 27-10-2020

DOI : 10.32508/stdjet.v3iSI3.680

\section{Copyright}

(c) VNU-HCM Press. This is an openaccess article distributed under the terms of the Creative Commons Attribution 4.0 International license.

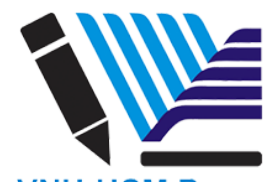

VNU-HCM Press

\begin{abstract}
Nowadays, transillumination imaging is more popular used in the medical field with the development of the vein finder application and also the non-invasive diagnosis applications. The less absorption of those main chromophores (melanin, oxy-hemoglobin, deoxy-hemoglobin, and water) of biological tissue within the near-infrared range $(700-1200 \mathrm{~nm})$ causes the relatively high transmission of the near-infrared light through biological tissue. They give us an "optical window" to seethrough the biological tissue non-invasively. Because of the high absorption of oxy-hemoglobin, deoxy-hemoglobin within the near-infrared range in comparison with these other parts in tissue, we can able to obtain a two dimensional (2D) transillumination image of the internal absorption structure such as blood vessel network, liver structure... in the body non-invasively. Using the lightemitting diode (LED)'s array to illuminate the human arm and using a low-cost camera to capture the image, we could obtain the blood vessel network image of the human arm. The captured image is blurred and difficult to distingue the structure inside. Because the camera captured the blood vessel network shadow that diffused on the skin surface. Professor Koichi Shimizu devised the depth-dependent point spread function (PSF) to describe the scattering effect from a light point source to the observation surface of a slab diffused medium by applying the diffusion approximation. By suppressing the scattering effect successfully, we could restore the clear image from the blurred image by de-convolution with the appropriated PSF. With our proposed technique, we could reconstruct the absorbing structures such as the kidney and liver in biological tissue. Using the restored images from different angles of view, we could reconstruct the cross-sectional images and three-dimensional image of the absorbing structures in an animal's abdomen. However, the depth information of the absorbing-structure was required in practice to calculate the appropriate PSF. Therefore, in order to make this method more practical, the depth information is crucial. In this paper, we proposed the novel techniques for estimating the parameters of absorbing structure (depth, center location, and width) in the turbid medium by using the convolution and deconvolution operations with the devised PSF. Firstly, by observing images with two-wavelength selected at which the absorption and scattering properties of the medium are different. While changing the depth step-by-step, two convoluted images were compared after convolving the captured image at one of the wavelengths with the PSF calculated by using the absorption and scattering coefficients from another wavelength. We can obtain the correct depth that gives the minimum difference between the two convoluted images. Secondly, we proposed the technique to estimate the depth information from a single transillumination image in order to make this method more practical. Finally, the absorbing structure image can be restored by suppressing the scattering effect. The proposed techniques were validated and examined not only in the simulation but also in the experiment.
\end{abstract}

Key words: transillumination imaging, scattering suppression, convolution, Point spread function (PSF), depth estimation

\section{INTRODUCTION}

The less absorption of those main chromophores (melanin, oxy-hemoglobin, deoxy-hemoglobin, and water) of biological tissue within the near-infrared (NIR) range $(700-1200 \mathrm{~nm})$ causes the relatively high transmission of the near-infrared light through biological tissue. They give us an "optical window" to see-through the biological tissue non-invasively.
Using the NIR transillumination imaging technique, it is possible to safely perform and visualize the surface vascular structure of the human body noninvasively ${ }^{1,2}$. The ab-normal tissue diagnosis, or veins location, and authentication applications using the transillumination image of the breast tissue, or the palm vein, and the finger vein are common nowadays. Because of the high absorption of oxy-hemoglobin, 
deoxy-hemoglobin within the near-infrared range in comparison with these other parts in tissue, we can able to obtain a two dimensional (2D) transillumination image of the internal absorption structure such as blood vessel network, liver structure... in the body non-invasively. Using the light-emitting diode (LED)'s array to illuminate the human arm and using a low-cost camera to capture the image, we could obtain the blood vessel network image of the human arm. The captured image is blurred and difficult to distingue the structure inside. Because the camera captured the blood vessel network shadow that diffused on the skin surface. The blurred effect increases while the absorbing-structure locates deeper in the medium. This is the major problem to cause misidentification in intravenous injection and personal authentication.

To overcome this problem, Professor Koichi Shimizu, who also contributed to this work, devised the depthdependent point spread function (PSF) to describe the scattering effect from a light point source to the observation surface of a slab diffused medium by applying the diffusion approximation to the radiative transport equation $^{2}$. By suppressing the scattering effect successfully, we could restore the clear image from the blurred image by de-convolution with the appropriated PSF. Using the restored images from different angles of view, we could reconstruct the cross-sectional images and three-dimensional image of the absorbing structures such as the kidney and part of liver in an animal's abdomen.

However, the depth information of the absorbingstructure and the optical properties of the medium were required in practice to calculate the appropriate PSF. Therefore, in order to make this method more practical, the depth information is crucial. In order to make this method more practical, the novel techniques for estimating the parameters of absorbing structure (depth, center location, and width) in the turbid medium by convolution and de-convolution with the point spread function (PSF) were devised. This paper presents the two novel techniques for the estimation depth information of the absorbing structure in the two-dimensional (2D) transillumination image. The proposed techniques were validated and examined not only in the simulation but also with the tissue-equivalent phantom.

\section{CATTERING SUPPRESSION TECHNIQUE}

Figure 1 shows the image formation of a black-painted disk in the clear medium and in the turbid medium.
The near-infrared light is illuminated from outside of the medium and goes through the absorbing structure, and projects the shadow on the surface of the turbid medium.

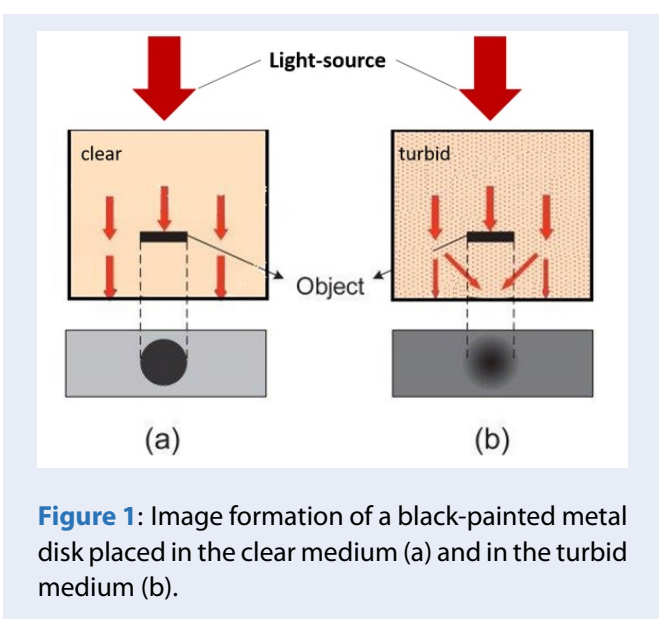

In the transillimiantion of a light-absorbing structure, the camera was obtained the light goes through the medium and captured the spatial light distribution.

As shown in Figure 1, the observed image captured the shadow appeared at the observation surface. In the observed image of the turbid medium, the blurred effect makes us cannot identify the structure and its size. Therefore, to understand the shape of the absorbing structure, scattering suppression is crucial.

The observed image of a light absorbing-structure in a turbid medium captured by the camera as shown in Figure 1 (b) can be described as the following general image formation equation'

$$
y=h \times x+n,
$$

where $y, h, x, n$ denote the observed image, the blurring point spread function (PSF), the original image, and the noise component. $\times$ denotes the convolution operation. In this research, the noise component was ignored, the Eq. (1) becomes

$$
y=h \times x .
$$

From Eq. (2), if we can describe the blurring effect by calculating an appropriate PSF, the clearer image of the absorbing-structure could be restored by deconvolving the observed image with the blurring PSF. The blurring PSF is difficult to obtained through the measurement. We cannot measure all PSFs for every condition in one observed image. Professor Koichi Shimizu, who also contributed to this work, was described theoretically the blurring PSF by approximating diffusion equation. 


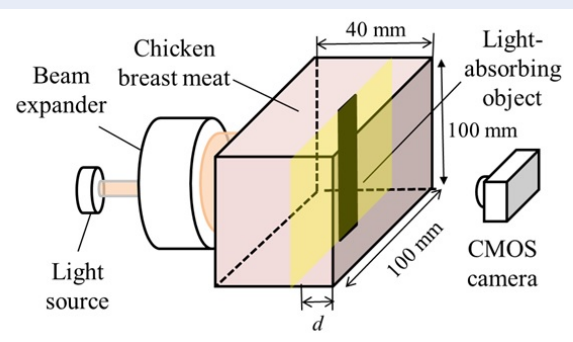

Figure 2: Typical experimental setup for lightabsorbing imaging

In a previous study ${ }^{2}$, Professor Koichi Shimizu considered a light point source placed in a slab turbid medium. The camera captured the spatial light distribution at the observation surface could be described as the depth-dependent point spread function (PSF). Because the spatial light distribution increases as the depth of the light point source increases. The spatial distribution of light intensity at the observation surface, described the blurring phenomena by scattering, is given by

$$
\begin{aligned}
& \operatorname{PSF}(\rho)=\frac{3 P_{0}}{(4 \pi)^{2}} \times\left\{\left(\mu_{s}^{\prime}+\mu_{a}\right)+\right. \\
& \left.\left[\kappa_{d}+\frac{1}{\left(\rho^{2}+d^{2}\right)^{1 / 2}}\right] \times \frac{d}{\left(\rho^{2}+d^{2}\right)^{1 / 2}}\right\} \\
& \times \frac{\exp \left[-\kappa_{d}\left(\rho^{2}+d^{2}\right)^{1 / 2}\right]}{\left(\rho^{2}+d^{2}\right)^{1 / 2}},
\end{aligned}
$$

where $\kappa_{d}^{2}=3 \mu_{a}\left(\mu_{s}^{\prime}+\mu_{a}\right)$; the $P_{0}, \mu_{s}^{\prime}, \mu_{a}, d$, and $\rho$ are the initial optical power of a point source, the reduced scattering coefficient, the absorption coefficient, the depth of a point source, and the radial distance in the cylindrical coordinate system, respectively.

The PSF calculated from Eq. (3) is the observed image of the spatial light distribution captured at the turbid medium surface. In contrast with the fluorescent imaging, the observed image in the light-absorbing imaging is the spatial shadow distribution. The light source in the light absorbing imaging was placed and illuminated the medium from outside. By reversing the observed image, the light absorbing-structure becomes the light source structure. By that way, we can apply the depth-dependent PSF of the light source in turbid medium calculated from Eq. (3) to de-blur the observed transillumination image of the absorbing structure. This consideration was validated and verified in simulation and experiment ${ }^{3,4}$. Figure 2 shows the typical experimental setup for transillumination imaging with biological tissue or turbid medium.
As shown in Figure 2, the black-painted metal object was used to make the ideally absorbing-structure. An acrylic container filled with tissue-equivalent medium or biological tissue was used to produce the diffused medium. The absorbing-structure was placed at a depth $d$ from the observation surface of the acrylic container. The homogenous light source was placed and illuminated the turbid medium from outside. The observed image of the light absorbingstructure is obtained using a camera. In this paper, the NIR light from a laser (Coherent Chameleon Ultra II Ti:Sapphire, 680 - $1080 \mathrm{~nm}$ tuneable wavelength $3.50 \mathrm{~W}$ ) through a beam expander was used to create the homogeneous illumination condition. The Complementary Metal Oxide Semiconductor (CMOS) camera (C11440-10C; Hamamatsu Photonics K.K.) was used to capture the transillumination image at high quality.

From the Eq. (2), by suppressing the scattering effect, the absorbing-structure $h_{a b s}$ could be restored as

$$
h_{a b s}=y \otimes x,
$$

where $\otimes$ denotes the deconvolution operation.

Figure 3 shows the observed image of a black-painted metal plate $(10.00 \mathrm{~mm} \times 100.00 \mathrm{~mm} \times 1.00 \mathrm{~mm})$ and the processed image using Eq. (4). The depth of the metal plated was $d=6.00 \mathrm{~mm}$ from the observation surface. The chicken breast meat filled into the container to produce the diffused medium $\left(\mu^{\prime}{ }_{s}\right.$ $\left.=1.00 / \mathrm{mm}, \mu_{a}=0.01 / \mathrm{mm}\right)$. The laser light source at $800 \mathrm{~nm}$ wavelength illuminated the medium from outside through a beam expander. Figure 3 (a), (b), and (c) are respectively show the restored image, the observed image, and the intensity profiles along the dashed lines on Figs. 3(a) and 3(b). The black line in Figure 3 (c) shows the original sizes of the light absorbing-structure. The measured widths of the absorbing-structure in the observed image and the restored image in terms of the Full-Width-HalfMaximum (FWHM) are $11.75 \mathrm{~mm}$ and $16.53 \mathrm{~mm}$, respectively. The true width of the absorbing-structure is $10.00 \mathrm{~mm}$. The result as shown in Figure 3 was confirmed the effectiveness of the scattering suppression technique using Eq. (4).

\section{ESTIMATION DEPTH INFORMATION OF THE STRUCTURE TECHNIQUES}

The blurring effect by diffusing could be described and effectively suppressed as mentioned above. As shown in Figure 3, the observed image contains only one absorbing-structure at a specific depth $d$. The restored image using Eq. (4) with the PSF calculated at 


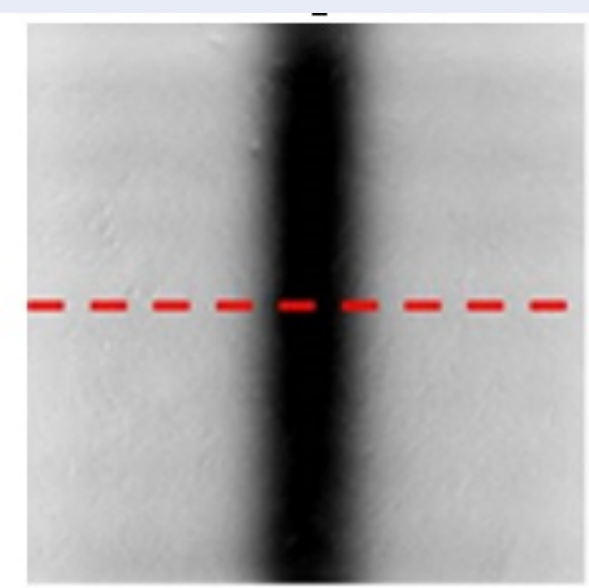

(a) Proposed

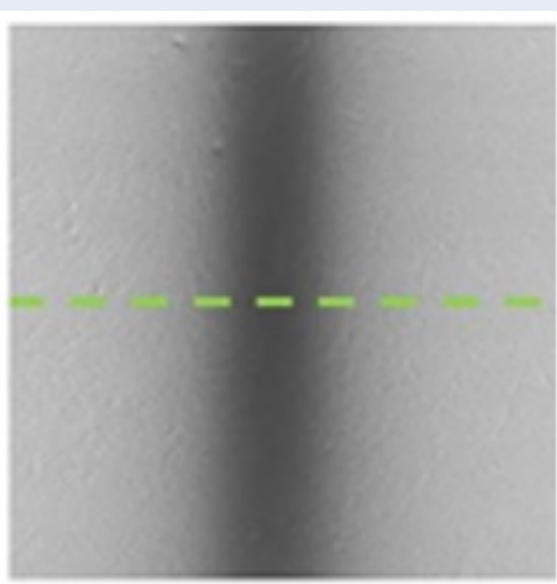

(b) Observed

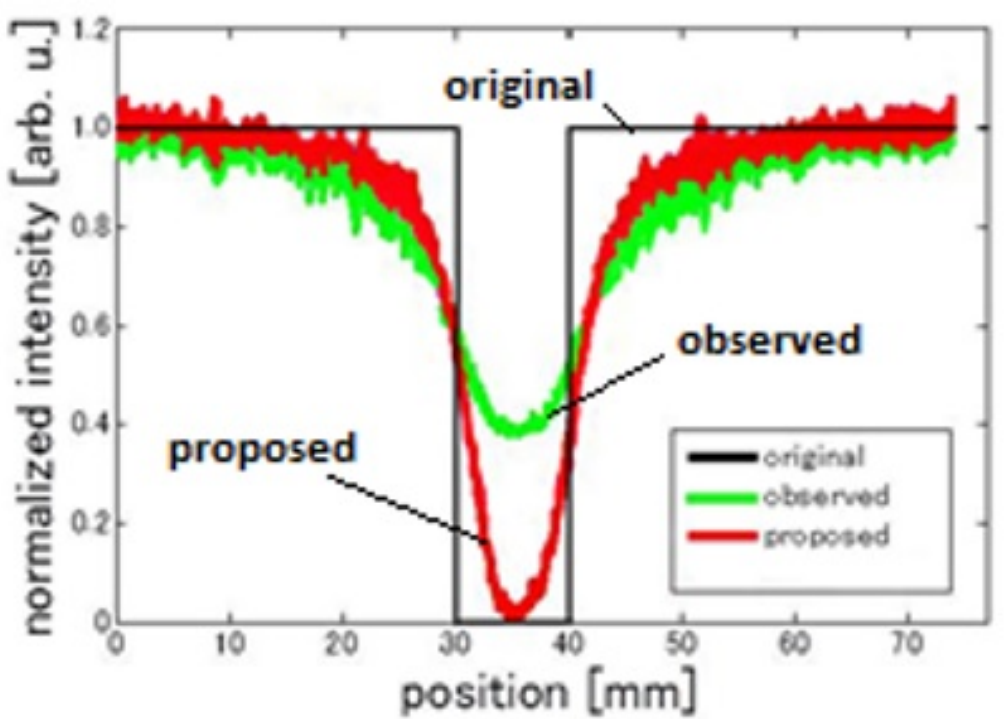

(c) Intensity

Figure 3: Result with light-absorbing imaging of a black-painted metal plate in chicken breast meat medium.

the known specific depth $d$ can be restored efficiently. However, in practice, the observed image usually does not contain only the simple absorbing-structure at a specific depth $d$. With the PSF calculated from Eq. (3) at a specific depth $d$, the part of the image that came from the depth $d$ can be restored correctly. In the image of a complex structure, such as the slant structure or blood vessel network, we could not restore the whole image by using one calculated PSF at a specific depth $d$. While a part of the image restored correctly, the other parts of the image are incorrect. They are generally smaller than the observed size and the true size of the absorbing-structure. In Eq. (3), we need to have the depth information, the optical properties of the medium, and the size of the observation surface to calculate the blurring PSF. Therefore, the depth information of the absorbing-structure is crucial to obtain the appropriate PSF in practice. In this paper, we devised the estimation techniques for estimating the depth information of the light absorbing-structure. In addition, one popular commercial application of transillumination imaging is the vein finder. The vein finder device observed the shadow of the vein network and projected back onto the skin. This may help 
the nurse or doctor to locate the vein by looking to the projected shadow on the skin. Even after the image processing, they cannot provide the information about blood vessel network, the depth and size of the blood vessel.

After have the depth information, we can de-blur the observed image and restore the shape and size of the absorbing-structure. From that, we can measure or estimate the width of the light absorbing-structure.

\section{Using two-wavelength perspective images}

The depth estimation technique using twowavelength perspective images based on the wavelength dependence of the optical characteristics in biological tissue. The observed images that are different by changing the wavelength of the light source. Two different wavelengths were selected at which the optical properties of the medium are different.

The observed images of a light-absorbing structure captured by illuminating the medium with wavelength $\lambda_{I}$ can be expressed by

$$
\operatorname{Ob}\left(\lambda_{i}, d\right)=\operatorname{Or} \times \operatorname{PSF}\left(\lambda_{i}, d\right),
$$

where $d, \times, \operatorname{Ob}\left(\lambda_{i}, d\right), \operatorname{Or}$, and $\operatorname{PSF}\left(\lambda_{i}, d\right)$, are respectively denote the depth of the absorbingstructure, the convolution operation, the observed image, the original light-absorbing structure, and the blurring PSF with medium characteristics of each wavelength. By calculating the blurring PSF from Eq. (3) with medium characteristics of each wavelength, and then convolving the observed image $O b\left(\lambda_{i}, d\right)$ with the calculated PSF for another wavelength, the new images will be compared and matched each other's, as given by

$$
\begin{aligned}
& O b\left(\lambda_{1}, d\right) \times \operatorname{PSF}\left(\lambda_{2}, d\right) \\
& =\operatorname{Ob}\left(\lambda_{2}, d\right) \times \operatorname{PSF}\left(\lambda_{1}, d\right),
\end{aligned}
$$

where $\times$ denotes the convolution operation.

By changing the depth $d$ for calculating the PSF and finding the matched pair as in Eq. (6), the depth estimation process can be described by

$$
\begin{aligned}
& \operatorname{Ob}\left(\lambda_{1}, d_{t}\right) \times \operatorname{PSF}\left(\lambda_{2}, d_{i}\right) \\
& =\operatorname{Or} \times \operatorname{PSF}\left(\lambda_{1}, d_{t}\right) \times \operatorname{PSF}\left(\lambda_{2}, d_{i}\right),
\end{aligned}
$$

and

$$
\begin{aligned}
& \operatorname{Ob}\left(\lambda_{2}, d_{t}\right) \times \operatorname{PSF}\left(\lambda_{1}, d_{i}\right) \\
& =\operatorname{Or} \times \operatorname{PSF}\left(\lambda_{2}, d_{t}\right) \times \operatorname{PSF}\left(\lambda_{1}, d_{i}\right),
\end{aligned}
$$

where $\times, d_{t}$, and $d_{i}$ are denote the convolution operation, the correct depth, and the estimated depth, respectively. The convoluted images will be matched when $d_{i}=d_{t}$
While changing the depth step-by-step, two convoluted images were compared after convolving the captured image at one of the wavelengths with the PSF calculated by using the absorption and scattering coefficients from another wavelength. We can obtain the correct depth that gives the minimum in terms RootMean-Square (RMS) difference between the two convoluted images from Eq. (7) and Eq. (8). Figure 4 shows an illustration of the process using Eq. (7) and Eq. (8).

\section{Using a single-wavelength observed image}

The depth estimation technique mentioned above using two-wavelength perspective images based on the wavelength dependence of the optical characteristics in biological tissue. However, due to the movement of the animal or human, the limitation of the imaging system, and the light illuminated condition, the proposed technique as mention above may have the limitation. To overcome these problems, we also propose another technique to estimate the depth information of the light-absorbing structure from the single-wavelength observed image. It will enlarge our estimation depth technique and make it be more practical.

As mentioned in section Using two-wavelength perspective images, with a PSF calculated from Eq. (3) at a specific depth $d$, we cannot restore the whole observed image of the complex light-absorbing structure. We propose a novel depth estimation technique from a single-wavelength observed image by following steps below:

Step 1: de-convolute the observed transillumination image using the PSF calculated from Eq. (3) with a specific depth $d_{i}$, ( $d_{i}$ varies from $0.1 \mathrm{~mm}$ to $\left.d_{\max }\right)$ :

$$
h_{a b s-i}=y \otimes x_{i}
$$

where $\otimes$ denotes the deconvolution operation.

Step 2: calculate the $y_{i}$ by convoluting $h_{a b s-i}$ obtained from Eq. (9) with the PSF calculated from Eq. (3) with a specific depth $d_{i}$, ( $d_{i}$ varies from $0.1 \mathrm{~mm}$ to $\left.d_{\max }\right)$ :

$$
y_{i}=h_{a b s-i} \times x_{i} ;
$$

where $\times$ denotes the convolution operation.

Step 3: compare in terms the correlation coefficient between the new convoluted image $y_{i}$ and the observed image $y$.

Figure 5 shows the illustration the step 1 of the depth estimation technique using a single-wavelength transillumination image. The deconvoluted image obtained from Eq. (9), as shown in Figure 5, will be convoluted with the PSF calculated from Eq. (3) with a 


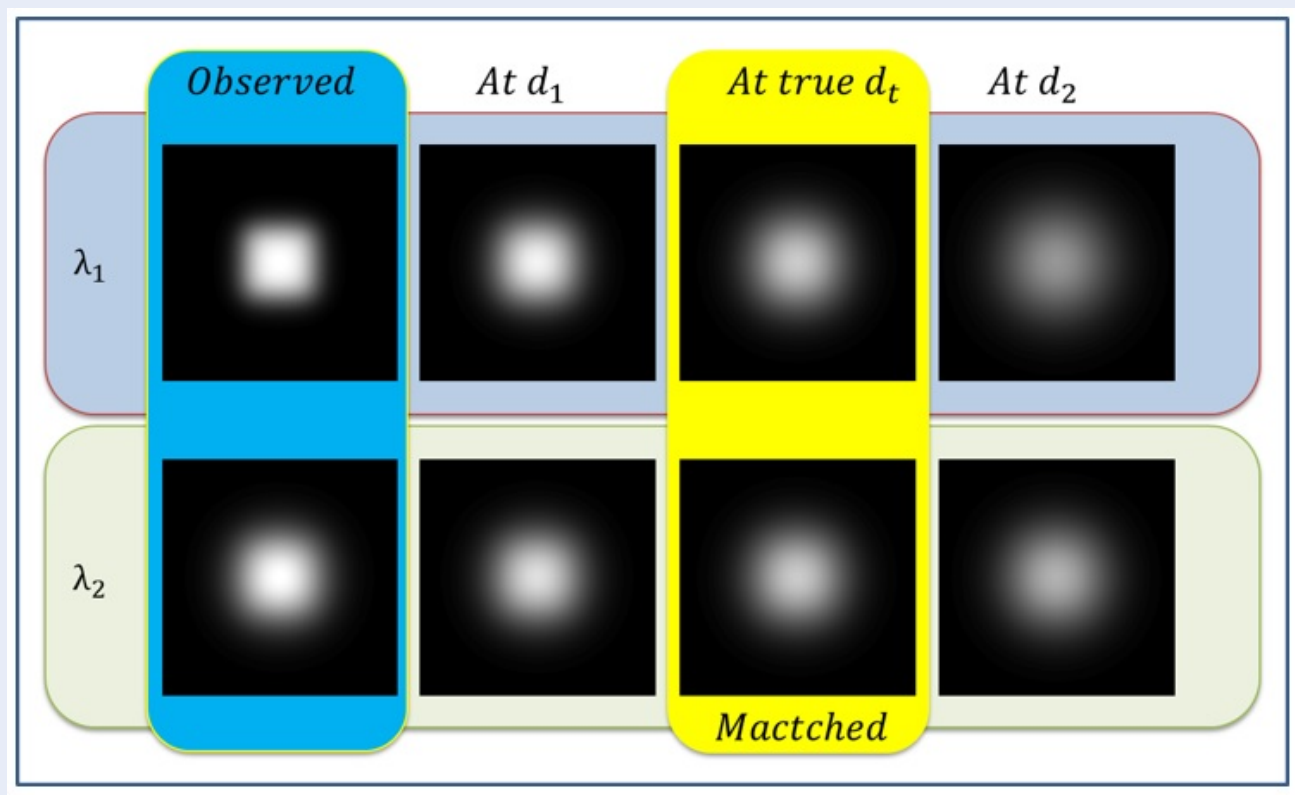

Figure 4: Illustration of the depth estimation technique using two-wavelength perspective images.

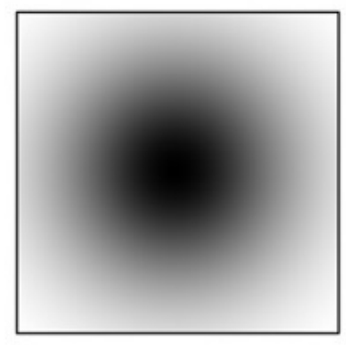

(区)
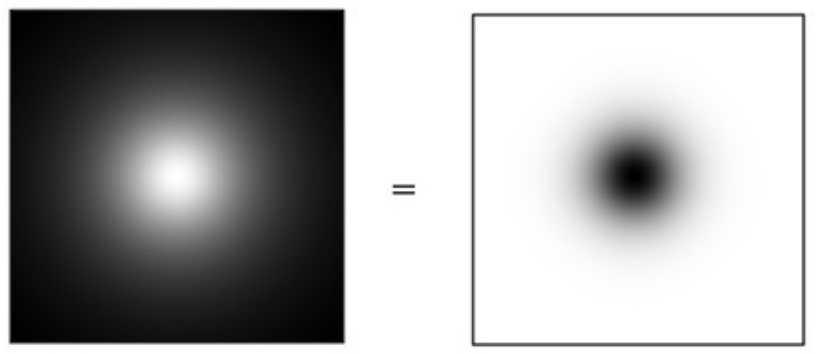

Figure 5: Illustration the step 1 of the depth estimation technique using single-wavelength transillumination image.

specific depth $d_{i}$, ( $d_{i}$ varies from $0.1 \mathrm{~mm}$ to $\left.d_{\max }\right)$. The process of step 2 was illustrated as shown in Figure 6. The estimated depth $d_{\text {est }}$ is the specific depth $d_{i}$ where the correlation coefficient got maximum value.

The technique mentioned in section Using twowavelength perspective images can combine with the technique mentioned in section Using a singlewavelength observed image to verify and improve the estimated result.

\section{VALIDATION IN SIMULATION}

To verify the proposed techniques, the validation of the proposed techniques was conducted in simulation.

\section{Light-absorbing object}

Figure 7 shows the light-absorbing squared metal plate placed in the transparent medium. A blackpainted squared plate $(10.00 \times 10.00 \mathrm{~mm})$ was simulated as an absorbing-structure and to be placed at the depth $d_{t}=5.00 \mathrm{~mm}$ from the observation surface. The observation surface of the diffused medium is considered as a squared surface $(100.00 \times 100.00 \mathrm{~mm})$.

The simulation conditions were made as close as possible to the experimental conditions as shown in Figure 2. The simulated images were obtained by convolving the original light-absorbing structure image as in Figure 7 with the corresponding PSF calculated from Eq. (3). The depth of the absorbing-structure varies from 1.00 to $10.00 \mathrm{~mm}$ with the interval 1.00 $m m$. 

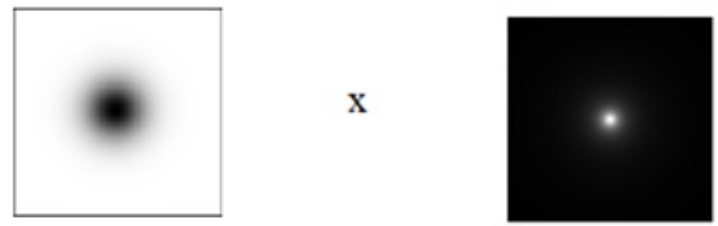

$=$

$\mathrm{x}$

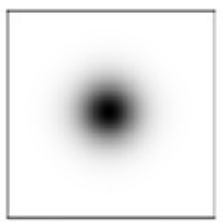

\section{$\mathrm{x}$}

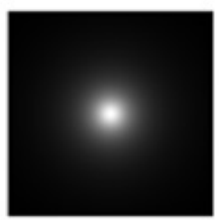

$=$

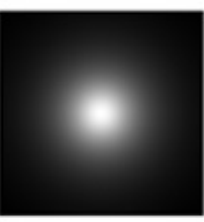

$=$

Figure 6: Illustration the step 2 of the depth estimation technique using single-wavelength transillumination image.

Figure 7: Image of the absorber obtained with transparent medium

\section{Using two-wavelength perspective images}

We simulated two models: the same scattering coefficients and the same absorption coefficients.

Model A: same scattering coefficient: $\mu_{s}^{\prime}\left(\lambda_{1}\right)=$ $\mu_{s}^{\prime}\left(\lambda_{2}\right)=1.00 / \mathrm{mm}, \mu_{a}\left(\lambda_{1}\right)=0.01 / \mathrm{mm}$, and $\mu_{a}\left(\lambda_{2}\right)=0.10 / \mathrm{mm}$.
Figure 8 shows the RMS difference for model $\mathrm{A}$ at each depth.

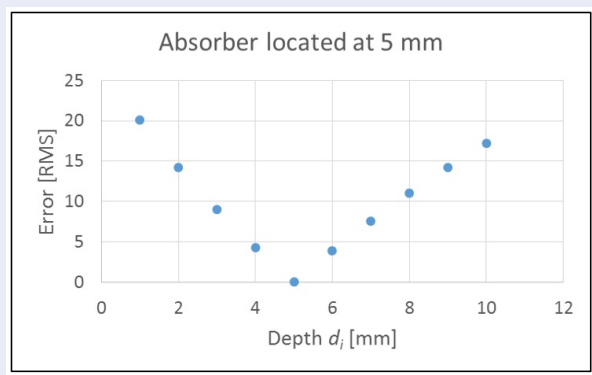

Figure 8: Estimation depth of the absorbingstructure in model $\mathrm{A}$. The RMS difference for model with the same scattering condition. The true depth is $5.00 \mathrm{~mm}$.

Model B: same absorption coefficient: $\mu_{a}\left(\lambda_{1}\right)=$ $\mu_{a}\left(\lambda_{2}\right)=0.005 / m m, \mu_{s}^{\prime}\left(\lambda_{1}\right)=0.85 / m m, \mu_{s}^{\prime}\left(\lambda_{2}\right)=$ $1.15 / \mathrm{mm}$.

Figure 9 shows the RMS difference for model B at each depth.

As shown in Figure 8 and Figure 9, the difference in terms of RMS became the minimum at the given cor- 


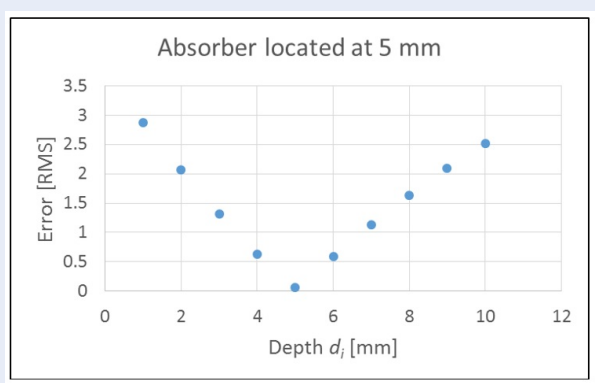

Figure 9: Estimation depth of the absorbingstructure in model B. The RMS difference for model with the same absorption condition. The true depth is $5.00 \mathrm{~mm}$.

rect depth.

\section{Using a single-wavelength observed image}

The model was made with the optical properties as following conditions: the scattering coefficient $\mu_{s}^{\prime}=1.00 / \mathrm{mm}$ and the absorption coefficient $\mu_{a}=$ $0.01 / \mathrm{mm}$. The correct depth was given with $d_{t}=5.00$ $\mathrm{mm}$.

Figure 10 shows the correlation coefficient calculated at each depth. As shown in Figure 10, at the true depth, the correlation coefficient became the maximum.

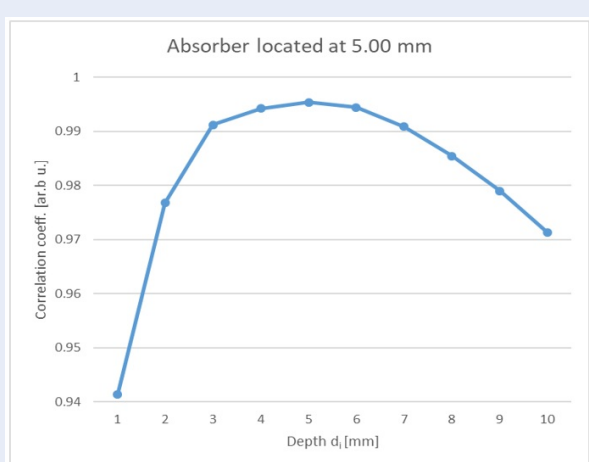

Figure 10: Estimation depth of the absorbingstructure using a single-wavelength observed image. The correct depth is $5.00 \mathrm{~mm}$.

The results suggest that the depth estimation technique mentioned above in section Using twowavelength perspective images and in section Using a single-wavelength observed image is valid. The results validated the feasibility of the proposed technique.

\section{VALIDATION IN EXPERIMENT WITH TISSUE-EQUIVALENT PHANTOM}

This section presents the validation of the proposed techniques in the experiment. Figure 11 shows the experimental setup for the experiment with a black squared plate in the tissue-equivalent phantom.

For easy changing the position of the absorbingstructure in the medium, the container, as shown in Figure 11, was filled by the Intralipid suspension (Fresenius Kabi AG), distilled water, and black ink (INK30-B; Pilot Corp.).

The black squared metal plate $(10.00 \mathrm{~mm} \times 10.00 \mathrm{~mm}$ $\times 1.00 \mathrm{~mm}$ ) was used as the light-absorbing structure. The depth was changed from $4.00 \mathrm{~mm}$ to $6.00 \mathrm{~mm}$. The wavelength of the NIR light source was selected at $800 \mathrm{~nm}$. For making the different conditions, the Intralipid suspension or the black ink was added for changing the optical properties of the medium.

\section{Using two-wavelength perspective image}

The experiments were conducted as same conditions with these models mentioned in section Using twowavelength perspective images. Model C: optical properties of scattering medium 1 and 2 were made as:

+ medium 1: $\mu_{s}^{\prime}(1)=1.00 / \mathrm{mm}$ and $\mu_{a}(1)=$ $0.01 / \mathrm{mm}$

+ medium 2: $\mu_{s}^{\prime}(2)=1.00 / \mathrm{mm}$ and $\mu_{a}(2)=$ $0.10 / \mathrm{mm}$.

The RMS difference was calculated at each depth. The result was shown in Figure 12 and Figure 13. Figure 12 shows the calculated result in terms of RMS with the correct depth is $4.00 \mathrm{~mm}$.

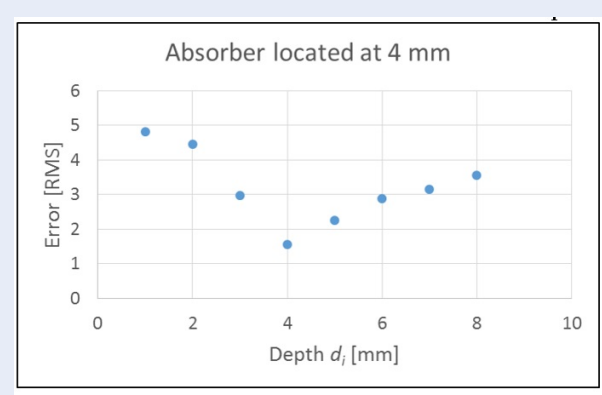

Figure 12: Estimation depth of the structure in model C. The correct depth is $4.00 \mathrm{~mm}$.

Figure 13 shows the calculated result in terms of RMS with the correct depth is $6.00 \mathrm{~mm}$.

As shown in Figure 12 and Figure 13, the difference in terms of RMS became the minimum at the given correct depth. 


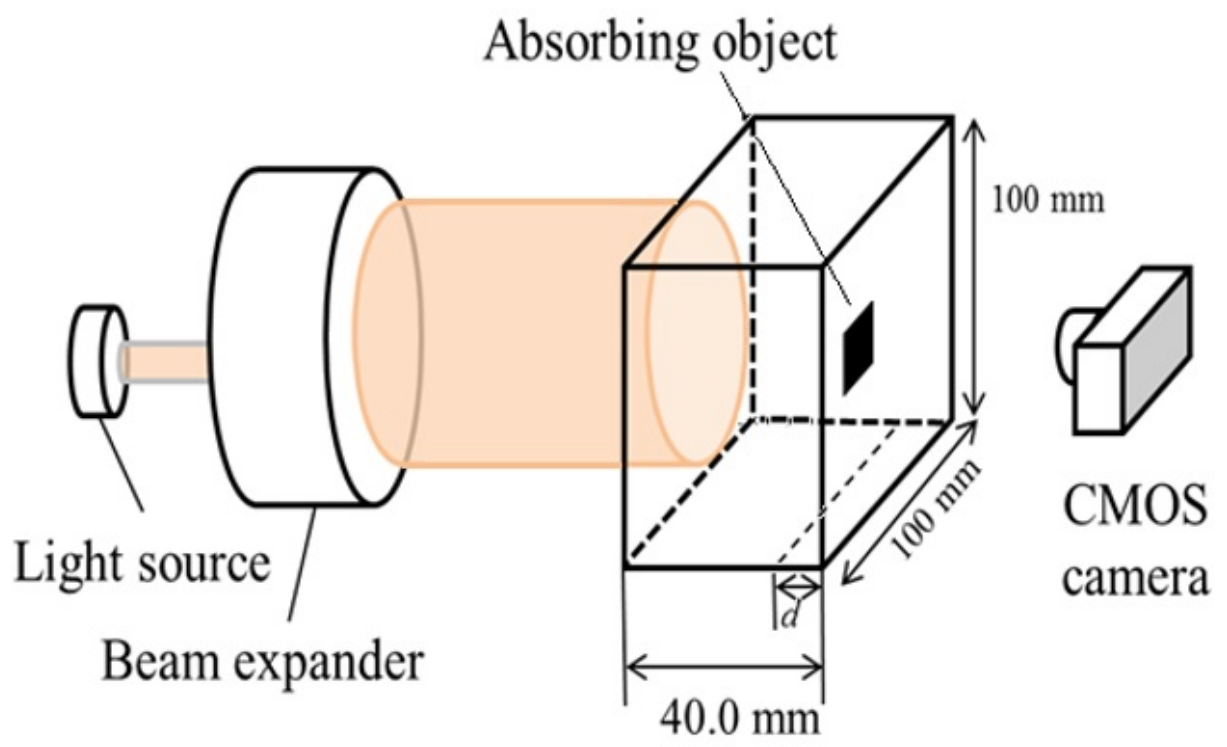

Figure 11: Experimental setup for the experiment with a black squared metal plate in the tissue-equivalent phantom.

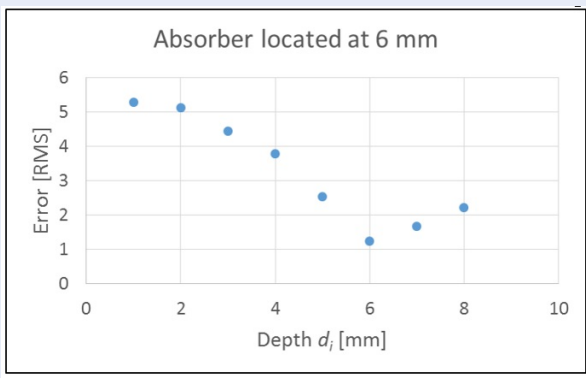

Figure 13: Estimation depth of the structure in model C. The correct depth is $6.00 \mathrm{~mm}$.

Model D: optical properties of scattering medium 1 and 2 were made as:

+ medium 1: $\mu_{s}^{\prime}(1)=0.80 / \mathrm{mm}$ and $\mu_{a}(1)=$ $0.05 / \mathrm{mm}$

+ medium 2: $\mu_{s}^{\prime}(2)=1.00 / \mathrm{mm}$ and $\mu_{a}(2)=$ $0.01 / \mathrm{mm}$.

Figure 14 and Figure 15 are respectively show the result with the given correct depth is $4.00 \mathrm{~mm}, 6.00 \mathrm{~mm}$. Figure 15 shows the result with the true depth is 6.00 $m m$.

As shown in Figure 14 and Figure 15, the difference in terms of RMS became the minimum at the given correct depth. The estimation depth is matched exactly with the given depth.

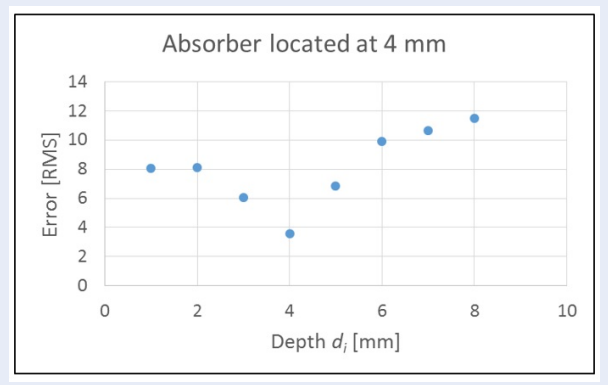

Figure 14: Estimation depth of the structure in model D. The correct depth is $4.00 \mathrm{~mm}$.

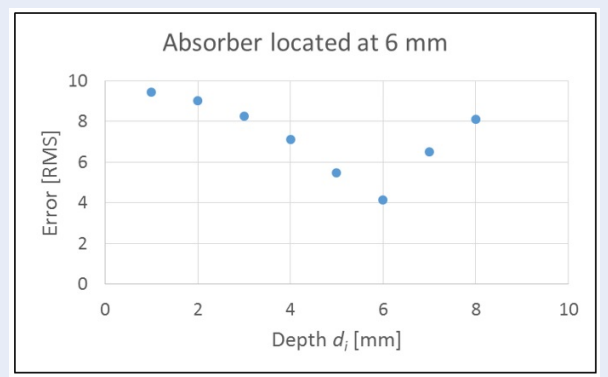

Figure 15: Estimation depth of the structure in model D. The correct depth is $6.00 \mathrm{~mm}$. 
Using a single-wavelength observed image

The experiment was conducted as same conditions with the model mentioned in section Using a singlewavelength observed image. The optical properties of scattering medium were made as: $\mu_{s}^{\prime}(1)=$ $1.00 / \mathrm{mm}$ and $\mu_{a}(1)=0.01 / \mathrm{mm}$. The absorbingstructure was placed at the depth $d_{t}=5.00 \mathrm{~mm}$ from the observation surface.

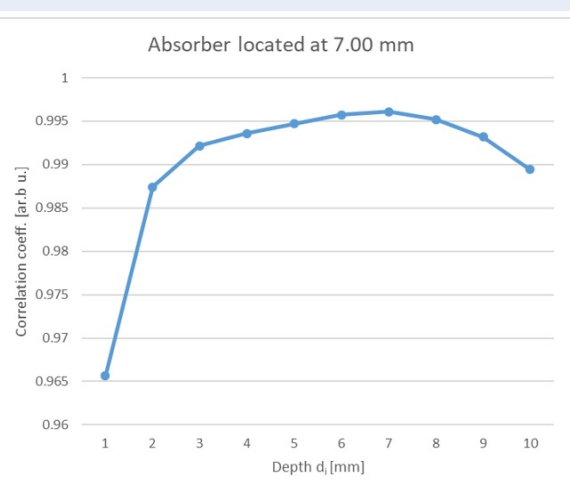

Figure 16: Estimation depth of the absorbingstructure using a single-wavelength observed image. The correct depth is $7.00 \mathrm{~mm}$.

Figure 16 shows the correlation coefficient calculated at each depth. As shown in Figure 16, the correlation coefficient became the maximum at the given correct depth.

The proposed technique was validated and examined in experiment with a tissue-equivalent phantom. The results shown that the proposed techniques in section Using two-wavelength perspective image and section Using a single-wavelength observed image can be applied in practice with a good condition of an imaging system.

\section{RESULTS AND DISCUSSION}

As shown in these figures in section Validation in simulation and section Validation in experiment with tissue-equivalent phantom, the estimated result is exactly matched with the given value. The results confirmed that the proposed depth-estimation techniques can be applied in practice with a good condition of an imaging system. We could able to estimate the depth information of the structure even with only single observed image. We also can combine both techniques mentioned in section Estimation depth information of the structure techniques to obtain better estimated depth result in practice.

Figure 17 shows the results of image restoration of the observed image while the absorbing-structure placed at a true depth of $6.00 \mathrm{~mm}$ using these results. For comparison, the restored images after de-convoluted with PSF at $d=4.00 \mathrm{~mm}$ and $8.00 \mathrm{~mm}$ were also shown. By obtaining the correct estimated depth, it can be seen that appropriate image restoration has been performed. Through such analysis, the usefulness of the proposed scattering-suppression and estimation depth techniques was confirmed.

\section{CONCLUSION}

With the view toward the realization of the transillumination imaging modality for authentication and diagnostic application, we proposed the scattering suppression technique and the novel depth estimation techniques to restore the clearer image and the "true shape" of the absorbing-structure.

By observing images with two-wavelength selected at which the scattering property of the medium is different. The transillumination image at one of the wavelengths is convolved with the calculated PSF with the optical properties of another wavelength while changing the depth. We can obtain the correct depth that gives the minimum difference between the two convoluted images.

By observing the image with a single-wavelength, we also can estimate the depth of the absorbing-structure. By combining these techniques, we can obtain an estimated depth value in agree with the given depth.

The proposed techniques were examined and validated in the simulation and also in the experiments with the tissue-equivalent phantom.

In future works, the proposed techniques will be examined with the image obtained with biological tissue.

The results in this paper suggested that even with a limited angle of view, we can obtain the transillumination image sufficiently clear and information of the light-absorbing structure. These techniques are useful for developing the noninvasive imaging of the lightabsorbing structure. It is useful for developing the diagnosis modality and the application in medical field.

\section{ACKNOWLEDGMENTS}

This presented research was supported by Ho Chi Minh City University of Technology, VNU-HCM under Grant T-KHUD-2018-83.

\section{CONFLICT OF INTEREST}

The authors declare no conflict of interest. 


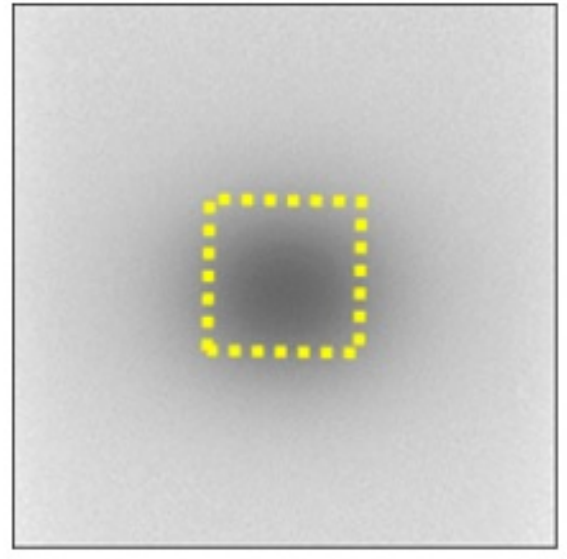

(a) observed image

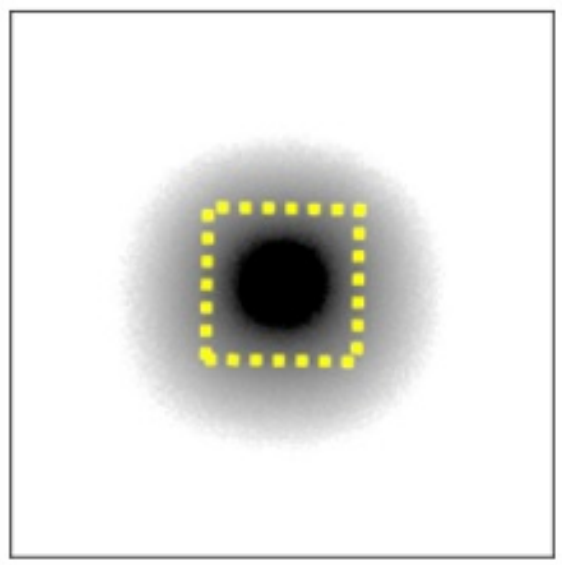

(c) restored with PSF $4.00 \mathrm{~mm}$

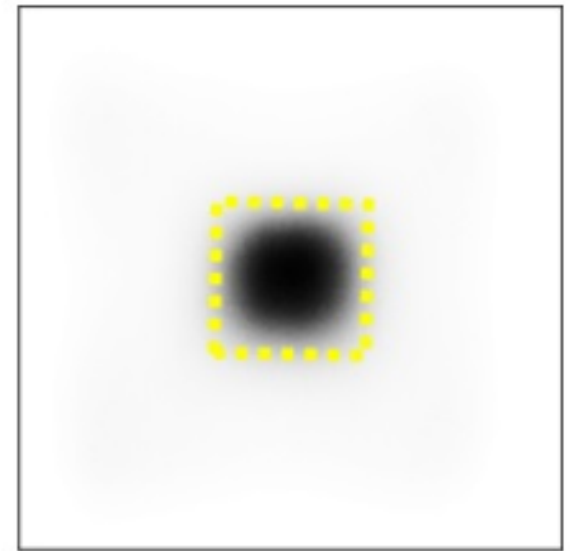

(b) restored with PSF $6.00 \mathrm{~mm}$

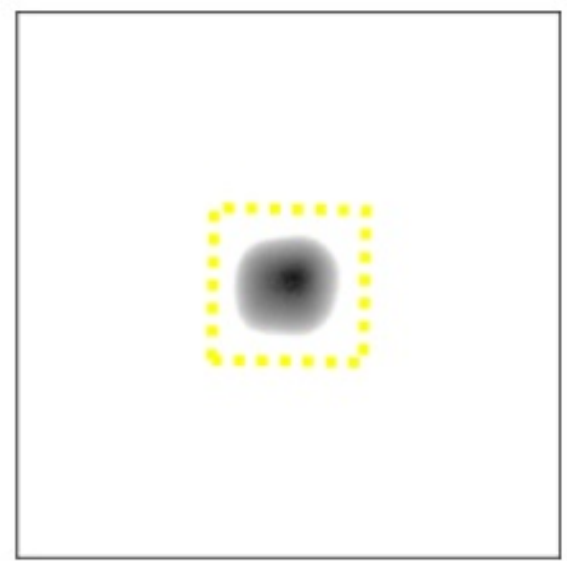

(d) restored with PSF $8.00 \mathrm{~mm}$

Figure 17: Effectiveness of scattering suppression using correctly estimated depth. The yellow dashed-line square represents the true size of the absorber.

\section{AUTHORS' CONTRIBUTIONS}

Conceptualization: Trung Nghia Tran, Takeshi Namita, and Koichi Shimizu.

Methodology: Trung Nghia Tran, Kohei Yamamoto, and Takeshi Namita.

Software: Trung Nghia Tran, Kohei Yamamoto, Ngoc An Dang Nguyen, and Minh Quang Nguyen.

Validation: Trung Nghia Tran, Kohei Yamamoto, Ngoc An Dang Nguyen, and To Ni Phan Van.

Data curation: Ngoc An Dang Nguyen, and To Ni Phan Van

Writing-original draft preparation: Ngoc An Dang Nguyen, and To Ni Phan Van.
Writing-review and editing: Anh Tu Tran, and Trung Nghia Tran.

Supervision: Trung Nghia Tran, Takeshi Namita, and Koichi Shimizu.

All authors have read and agreed to the published version of the manuscript.

\section{REFERENCES}

1. Tobisawa N, Namita T, Kato Y, Shimizu K. Injection Assist System with Surface and Transillumination Images. 5th International Conference on Bioinformatics and Biomedical Engineering, Wuhan. 2011;p. 1-4. Available from: https://doi.org/10. 1109/icbbe.2011.5780365. 
2. Shimizu K, Tochio K, Kato $\mathrm{Y}$. Improvement of transcutaneous fluorescent images with a depth-dependent point-spread function. Appl Opt. 2005;44:2154-2161. PMID: 15835361. Available from: https://doi.org/10.1364/AO.44.002154.

3. Tran TN, Yamamoto K, Namita T, Kato Y, Shimizu K. Threedimensional transillumination image reconstruction for small animal with new scattering suppression technique. Biomed Opt Express. 2014;5(5):1321-1335. PMID: 24876998. Available from: https://doi.org/10.1364/BOE.5.001321.

4. Tran TN, Van TNP, Tran AT, Yamamoto K, Namita T, Shimizu $K$, et al. Near -infrared transillumination imaging of veins using low -cost camera and scattering suppression - validation of practicality of developed system. Journal of Development Science and Technology. 2017;20(K3/2017):67-73. Available from: https://doi.org/10.32508/stdj.v20iK3.1094. 